\title{
Zitterbewegung and its effects on electrons in semiconductors
}

\author{
Wlodek Zawadzki \\ Institute of Physics, Polish Academy of Sciences \\ Al.Lotnikow 32/46, 02-668 Warsaw, Poland
}

(Dated: October 28, 2018)

\begin{abstract}
An analogy between the band structure of narrow gap semiconductors and the Dirac equation for relativistic electrons in vacuum is used to demonstrate that semiconductor electrons experience a Zitterbewegung (trembling motion). Its frequency is $\omega_{Z} \approx \mathcal{E}_{g} / \hbar$ and its amplitude is $\lambda_{Z}$, where $\lambda_{Z}=\hbar / m_{0}^{*} u$ corresponds to the Compton wavelength in vacuum $\left(\mathcal{E}_{g}\right.$ is the energy gap, $m_{0}^{*}$ is the effective mass and $u \approx 1.3 \times 10^{8} \mathrm{~cm} / \mathrm{sec}$ ). Once the electrons are described by a two-component spinor for a specific energy band there is no Zitterbewegung but the electrons should be treated as extended objects of size $\lambda_{Z}$. Possible consequences of the above predictions are indicated.
\end{abstract}

PACS numbers: 03.65.Pm 71.20.Nr 73.21.Fg

It was noted in the past that the $E(\mathbf{k})$ relation between the energy $E$ and the wavenumber $\mathbf{k}$ for electrons in narrow-gap semiconductors (NGS) is analogous to that for relativistic electrons in vacuum [1-4]. The analogy was also shown to hold for the presence of external fields which was experimentally confirmed on InSb [5]. This "semirelativity in semiconductors" is valid for time dependent phenomena as well, so that the cyclotron resonance of conduction electrons in InSb could be interpreted in terms of the time dilatation between a moving electron and an observer [5]. The semirelativistic phenomena appear at electron velocities of $10^{7}-10^{8} \mathrm{~cm} / \mathrm{sec}$, much lower than the light velocity $c$. The reason for this is that the maximum velocity $u$ in semiconductors, which plays the role of $c$ in vacuum, is about $10^{8} \mathrm{~cm} / \mathrm{sec}$.

Until present the semirelativistic considerations for semiconductors were concerned with phenomena related mostly to classical mechanics. The purpose of this contribution is to investigate the quantum domain described by the Hamiltonian for energy bands in NGS, which bears close similarity to the Hamiltonian for relativistic electrons in vacuum. The effects we predict should be much more readily observable in NGS than in vacuum so this investigation is of interest not only for the solid state physics but also for the high energy physics.

We begin with the $\mathbf{k} \cdot \mathbf{p}$ approach to InSb-type III-V semiconductor compounds, first written down by Kane [6]. Taking the limit of large spin-orbit energy $\Delta$, the resulting dispersion relation for the conduction and the light-hole bands is $E= \pm E_{p}$, where

$$
E_{p}=\left[\left(\frac{\mathcal{E}_{g}}{2}\right)^{2}+\mathcal{E}_{g} \frac{p^{2}}{2 m_{0}^{*}}\right]^{1 / 2} .
$$

Here $\mathcal{E}_{g}$ is the energy gap and $m_{0}^{*}$ is the effective mass at the band edge. This expression is identical with the relativistic relation for electrons in vacuum, with the correspondence: $\mathcal{E}_{g} \rightarrow 2 m_{0} c^{2}$ and $m_{0}^{*} \rightarrow m_{0}$. The electron velocity $\boldsymbol{v}$ in the conduction band described by Eq.(1) reaches the saturation value as $p$ increases. This can be seen directly by calculating $v_{i}=\partial E_{p} / \partial p_{i}$ and taking the limit of large $p_{i}$, or by using the analogy: $c=\left(2 m_{0} c^{2} / 2 m_{0}\right)^{1 / 2} \rightarrow\left(\mathcal{E}_{g} / 2 m_{0}^{*}\right)^{1 / 2}=u$. Taking the experimental parameters $\mathcal{E}_{g}$ and $m_{0}^{*}$ we calculate very similar value of $u \approx 1.3 \times 10^{8} \mathrm{~cm} / \mathrm{sec}$ for different III-V compounds.

Now we define an important quantity

$$
\lambda_{Z}=\frac{\hbar}{m_{0}^{*} u},
$$

which we call the length of Zitterbewegung for reasons given below. Here we note that it corresponds to the Compton wavelength $\lambda_{c}=\hbar / m_{0} c$ for electrons in vacuum. Let us suppose that we confine an electron to the dimensions $\Delta z \approx \lambda_{Z} / 2$. Then the uncertainty of momentum is $\Delta p_{z} \geq \hbar / \Delta z$ and the resulting uncertainty of energy $\Delta E \approx\left(\Delta p_{z}\right)^{2} / 2 m_{0}^{*}$ becomes

$$
\Delta E \geq 2 m_{0}^{*} u^{2}=\mathcal{E}_{g}
$$

Thus the electron confined to $\Delta z \approx \lambda_{Z} / 2$ has the uncertainty of energy larger than the gap, so that it "does not know" whether it belongs to the conduction or to the valence band.

Next we consider the band Hamiltonian for NGS. The momentum $\hat{\boldsymbol{p}}$ must be kept in operator form, so this Hamiltonian is not identical with the $\mathbf{k} \cdot \mathbf{p}$ scheme of Kane's. Still, it is derived within the same model including $\Gamma_{6}$ (conduction), $\Gamma_{8}$ (light and heavy hole) and $\Gamma_{7}$ (split-off) bands and it represents an $8 \times 8$ operator matrix [7]. We assume, as before, $\Delta \gg \mathcal{E}_{g}$ and omit the free electron terms since they are negligible for NGS. The resulting $6 \times 6$ Hamiltonian has $\pm \mathcal{E}_{g} / 2$ terms on the diagonal and linear $\hat{p}_{i}$ terms off the diagonal, just like in the Dirac equation for free electrons. However, the three $6 \mathrm{x} 6$ matrices $\hat{\alpha}_{i}$ multiplying the momentum components $\hat{p}_{i}$ do not have the properties of $4 \times 4$ Dirac matrices, which considerably complicates calculations. For this reason, with only a slight loss of generality, we take $\hat{p}_{z} \neq 0$ and $\hat{p}_{x}=\hat{p}_{y}=0$. In $\hat{\alpha}_{3}$ matrix, two rows and columns corresponding to the heavy holes contain only zeros and they can be omitted. The remaining Hamiltonian for the con- 
duction and the light hole bands reads

$$
\hat{H}=u \hat{\alpha}_{3} \hat{p}_{z}+\frac{1}{2} \mathcal{E}_{g} \hat{\beta},
$$

where $\hat{\alpha}_{3}$ and $\hat{\beta}$ are the well known $4 \times 4$ Dirac matrices [8]. The Hamiltonian (4) has the form appearing in the Dirac equation and in the following we can use the procedures of relativistic quantum mechanics (RQM).

The electron velocity is $\dot{z}=(1 / i \hbar)[z, \hat{H}]=u \hat{\alpha}_{3}$. The eigenvalues of $\hat{\alpha}_{3}$ are \pm 1 , so that the eigenvalues of $\dot{z}$ are, paradoxically, $\pm u$. In order to determine $\hat{\alpha}_{3}(t)$ we calculate $\dot{\hat{\alpha}}_{3}(t)$ by commuting $\hat{\alpha}_{3}$ with $\hat{H}$ and integrating the result with respect to time. This gives $\dot{z}(t)$ and we calculate $z(t)$ integrating again. The final result is (cf. Refs [9-11])

$$
z(t)=z(0)+\frac{u^{2} p_{z}}{E_{p}} t+A_{0} \frac{i \hbar u}{2 E_{p}} \exp \left(\frac{-2 i E_{p} t}{\hbar}\right),
$$

where $A_{0}=\hat{\alpha}_{3}(0)-u p_{z} / E_{p}$, and $E_{p}$ is given by Eq.(1) with $p=p_{z}$. The first two terms of Eq.(5) represent the classical electron motion, $u^{2} p_{z} / E_{p}$ being the classical constant velocity. The third term describes time dependent oscillations with a frequency of $\omega_{Z} \approx \mathcal{E}_{g} / \hbar$. Since $A_{0} \approx 1$, the amplitude of oscillations is $\hbar u / 2 E_{p} \approx$ $\hbar / 2 m_{0}^{*} u=\lambda_{Z} / 2$. In RQM the analogous oscillations are called Zitterbewegung (ZB, trembling motion), which explains the name given above to $\lambda_{Z}$. We note that the ZB does not obey Newton's first law since we have a nonconstant velocity without a force. In RQM it is demonstrated that the ZB is a result of interference between states of positive and negative electron energies.

In order to investigate the case of a definite sign of energy, we expand the multiband Hamiltonian of Ref [7] into an effective one-band Hamiltonian for the $\Gamma_{6}$ conduction band (here we assume quite generally $\hat{\mathbf{p}} \neq 0$ ). We do this by solving the initial set of six equations for the envelope functions (for $\Delta \gg \mathcal{E}_{g}$ ) by substitution, i.e. expressing the valence functions $f_{3} \ldots f_{6}$ by the conduction functions $f_{1}$ and $f_{2}$ for spin-up and spin-down states, respectively. When doing this we account for the changed normalization condition (see $[12,13])$. The final result is

$$
\begin{aligned}
\hat{H}=\frac{\mathcal{E}_{g}}{2} & +V+\frac{P^{2}}{2 m_{0}^{*}}+\frac{g^{*} \mu_{B}}{2} \boldsymbol{\sigma} \cdot \boldsymbol{B}-\frac{1}{\mathcal{E}_{g}}\left(\frac{P^{2}}{2 m_{0}^{*}}+\frac{g^{*} \mu_{B}}{2} \boldsymbol{\sigma} \cdot \boldsymbol{B}\right)^{2}+ \\
& +\frac{\hbar}{4 \mathcal{E}_{g} m_{0}^{*}} \boldsymbol{\sigma} \cdot(\boldsymbol{\nabla} V \times \boldsymbol{P})+\frac{\hbar^{2}}{4 \mathcal{E}_{g} m_{0}^{*}} \nabla^{2} V
\end{aligned}
$$

where $\mathbf{P}=\mathbf{p}+e \mathbf{A}$ is the canonical momentum, $V$ is an external potential, $\boldsymbol{\sigma}$ is the Pauli spin operator, $\mu_{B}$ is the Bohr magneton, $\mathbf{B}$ is an external magnetic field, and $g^{*}$ is the Landé spin factor. The first four terms represent the effective Pauli equation, the fifth is a nonparabolic correction to the energy, the sixth is an effective spinorbit interaction, and the last one is the effective Darwin term [14]. The Hamiltonian (6) is in relativistic analogy to $v^{2} / c^{2}$ expansion of the Dirac equation, known from RQM (see [15]). In our case this expansion is of the order $v^{2} / u^{2}$.

The effective Darwin term can be interpreted in terms of ZB. If an electron oscillates around the position $\mathbf{r}$, the potential energy can be expanded as follows: $V(\mathbf{r}+$ $\Delta \mathbf{r}) \approx V(\mathbf{r})+\Delta \mathbf{r} \cdot \boldsymbol{\nabla} V+(\Delta \mathbf{r} \cdot \boldsymbol{\nabla})(\Delta \mathbf{r} \cdot \boldsymbol{\nabla}) V / 2$. On average the odd powers of $\Delta \mathbf{r}$ vanish and the last term is $(\Delta \mathbf{r})^{2} \nabla^{2} V / 6$. It becomes equal to the effective Darwin term in Eq.(6) if $(\Delta \mathbf{r})^{2}=(3 / 4) \lambda_{Z}^{2}$, which corresponds to $\Delta z=\lambda_{Z} / 2$. This is in agreement with our previous arguments. However, once the electron is described by a two-component spinor for a specific energy band, as in Eq.(6), the quantity $\Delta \mathbf{r}$ is not the amplitude of $\mathrm{ZB}$ anymore. We discuss this below.

The four-component wave functions resulting from Eq.(4) can be transformed exactly into two-component functions for positive (or negative) electron energies. For free electrons this is done by applying the FoldyWouthuysen (FW) unitary transformation [16]. In our one-dimensional treatment the transformation reads

$$
e^{i S}=\frac{E_{p}+\hat{\beta} \hat{H}}{\left[2 E_{p}\left(E_{p}+m_{0}^{*} u^{2}\right)\right]^{1 / 2}} .
$$

The transformed wave functions $\Psi^{\prime}(z)$ are obtained from the initial functions $\Psi\left(z^{\prime}\right)$ as follows

$$
\Psi^{\prime}(z)=\int K\left(z, z^{\prime}\right) \Psi\left(z^{\prime}\right) d z^{\prime}
$$

where

$$
\begin{gathered}
K\left(z, z^{\prime}\right)=\frac{1}{2 \pi} \int\left(\frac{2 E_{p^{\prime}}}{E_{p^{\prime}}+m_{0}^{*} u^{2}}\right)^{1 / 2} \frac{1}{2}\left(1+\frac{\beta \hat{H}}{E_{p^{\prime}}}\right) \times \\
\exp \left[\frac{i p_{3}^{\prime}\left(z-z^{\prime}\right)}{\hbar}\right] d p_{3}^{\prime} .
\end{gathered}
$$

The kernel $K\left(z, z^{\prime}\right)$ is not a point transformation. Suppose we are interested in the eigenfunction of position $\hat{z}$. In the initial representation this function is $\Psi\left(z^{\prime}\right)=$ $\delta\left(z^{\prime}-z_{0}\right)$, and it follows from Eq.(8) that the transformed function is $K\left(z, z_{0}\right)$. The integral of $K\left(z, z_{0}\right)$ over $Z=z-z_{0}$ axis is unity. To get an idea of the extension of $K\left(z, z_{0}\right)$, we calculate its second moment and after some manipulations we obtain (see Ref. [17])

$$
\int Z^{2} K\left(z, z_{0}\right) d Z=\frac{1}{4} \lambda_{Z}^{2}
$$

Thus the extension of the transformed eigenfunction of position is $\left|z-z_{0}\right| \approx \lambda_{Z} / 2$. One can show that in the transformed state there is no Zitterbewegung since the FW transformation eliminates the negative energy components of the wave functions [11]. All in all, following the interpretation established in RQM, we are confronted with the following choice. 1) We deal with a point-like 
electron described by a four-component function, which experiences the ZB with the radius of $\lambda_{Z} / 2$.2) The electron is described by a two-component spinor for either positive or negative energy and there is no $\mathrm{ZB}$, but the electron is smeared to an object of the radius $\lambda_{Z} / 2$. It is this smearing that enters into the effective Darwin term in Eq.(6), as this equation describes electrons using twocomponent spinors for positive energies. In other words, the Darwin term is a direct consequence of smearing.

The above result has far reaching consequences. In most cases electrons in a specific band are described by effective band parameters obtained with the use of the Luttinger-Kohn (LK) transformation [18]. In fact, our Eq.(6) can also be obtained by this method. The LK transformation separates energy bands and it corresponds directly to the Foldy-Wouthuysen transformation in RQM. For the FW transformation the expansion parameter is $S_{F W}=p / 2 m_{0} c=k \lambda_{c} / 2$, while for the LK transformation it is $S_{L K}=\hbar k /\left(2 m_{0}^{*} \mathcal{E}_{g}\right)^{1 / 2}=k \lambda_{Z} / 2$. Once again we find the correspondence between $\lambda_{c}$ and $\lambda_{Z}$. It follows from the above reasoning that electrons in a given energy band described by the one-band effective mass approximation are not point particles, but rather we should attribute to them the size $\lambda_{Z}$.

Clearly, the magnitude of $\lambda_{Z}$ is essential. There is $\lambda_{Z}=\lambda_{c}(c / u)\left(m_{0} / m_{0}^{*}\right) \approx 0.89\left(m_{0} / m_{0}^{*}\right) \AA$ since, as mentioned above, $u \approx 1.3 \times 10^{8} \mathrm{~cm} / \mathrm{sec}$ for various materials. We estimate: for $\operatorname{GaAs}\left(m_{0}^{*} \approx 0.067 m_{0}\right) \lambda_{Z} \approx 13$ $\AA$, for $\operatorname{InAs}\left(m_{0}^{*} \approx 0.024 m_{0}\right) \lambda_{Z} \approx 37 \AA$, for $\mathrm{InSb}$ $\left(m_{0}^{*} \approx 0.014 m_{0}\right) \lambda_{Z} \approx 64 \AA$. Thus, in contrast to vacuum $\left(\lambda_{c}=3.86 \times 10^{-3} \AA\right)$ the length of ZB in semiconductors can be quite large and one can confine electrons to the dimensions of $\lambda_{Z}$ using quantum wells, wires, dots, or external fields. For an electron in a magnetic field the magnetic radius is $L=(\hbar / e B)^{1 / 2} \approx 80 \AA$ at $B=10 \mathrm{~T}$, and it is fairly easy to achieve $L<\lambda_{Z}$ for NGS.

It may appear unreasonable to ascribe to an electron the size of, say, $70 \AA$. However, it is well known that the values of $1 / m_{0}^{*}$ and $g_{0}^{*}$ in very narrow gap semiconductors can be 100 times larger than the corresponding values for free electrons. The special property of $\lambda_{Z}=\hbar / m_{0}^{*} u$ is that it cumulates the largeness of $1 / m_{0}^{*}$ and of $1 / u$ for semiconductors, as compared to $1 / m_{0}$ and $1 / c$ for vacuum. We emphasize again that the form of the Darwin term in Eq.(6) corresponds to the electron size of $\lambda_{Z}$.

The Zitterbewegung length $\lambda_{Z}$ appears in the description of interband tunneling. The tunneling in vacuum from the Dirac sea of electrons with negative energies to the empty states of electrons with positive energies in the presence of a linear potential $V(z)=e F z$ has been treated by RQM $[19,20]$. The tunneling probability is proportional to $\exp (-W)$, where $W=\pi m_{0}^{2} c^{2} / e \hbar F=$ $(\pi / 2)\left(z_{0} / \lambda_{c}\right)$, in which $z_{0}=2 m_{0} c^{2} / e F$ is the tunneling length. This probability becomes appreciable at critical electric fields $F_{c r}$ such, that $z_{0} / \lambda_{c} \approx 1$, which gives $F_{c r}=$ $2 m_{0}^{2} c^{3} / e \hbar \approx 2.6 \times 10^{16} \mathrm{~V} / \mathrm{cm}$. Fields of this intensity are not available in terrestrial conditions. For semiconductors the corresponding probability of tunneling between

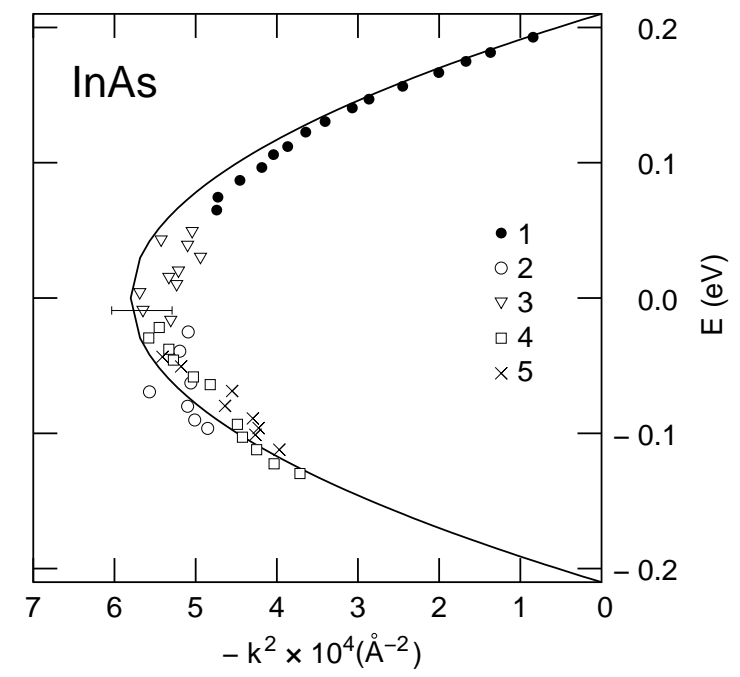

FIG. 1: Energy-wave vector dependence in the forbidden gap of InAs. Various symbols show experimental data of Parker and Mead [22] for five InAs samples, the solid line is theoretical fit using Eq.(11). The determined parameters are $\lambda_{Z}=$ $41.5 \AA$ and $u=1.33 \times 10^{8} \mathrm{~cm} / \mathrm{sec}$.

the light-hole and the conduction bands is described by the exponential factor $W=\pi \mathcal{E}_{g}^{3 / 2} m_{0}^{* 1 / 2} / 2 \sqrt{2} e \hbar F=$ $(\pi / 2)\left(z_{0} / \lambda_{Z}\right)$, where $z_{0}=\mathcal{E}_{g} / e F$ is the corresponding tunneling length [21]. Now the critical field, at which $z_{0} / \lambda_{Z} \approx 1$, is $F_{Z}=\mathcal{E}_{g}^{3 / 2} m_{0}^{* 1 / 2} / \sqrt{2} e \hbar$. This is of the order of $10^{5} \mathrm{~V} / \mathrm{cm}$, which agrees with the field intensities used in semiconductor tunnel diodes.

In fact, $\lambda_{Z}$ can be measured directly. We write Eq.(1) in the form

$$
E= \pm \hbar u\left(\lambda_{Z}^{-2}+k^{2}\right)^{1 / 2}
$$

For $k^{2}>0$ this formula describes the conduction and the light-hole bands. But for imaginary values of $k$ there is $k^{2}<0$ and Eq.(11) describes the dispersion in the energy gap. This region is classically forbidden but it can become accessible through quantum tunneling. Figure 1 shows the data for the dispersion in the gap of InAs, obtained by Parker and Mead [22] from tunneling experiments with double Schottky barriers. The solid line indicates the fit using Eq.(11). The value of $\lambda_{Z}$ is determined directly by $k_{0}$ for which the energy is zero: $\lambda_{Z}^{-2}=k_{0}^{2}$. The fit gives $\lambda_{Z} \approx 41.5 \AA$ and $u \approx 1.33 \times 10^{8}$ $\mathrm{cm} / \mathrm{sec}$, in good agreement with the above estimation for InAs. Similar data for GaAs give $\lambda_{Z}$ between $10 \AA$ [23] and $13 \AA$ [24], again in good agreement with the above estimation.

Our results should lead to two categories of observable effects. The first is related to the final electron size. For example, electron-electron interaction at short distances or electron interaction with short wavelength phonons should be affected by the final size (see [25]). The effective Darwin term belongs to this group. The second category is associated with the question: what happens 
when electrons are confined to dimensions smaller than $\lambda_{Z}$ ? It is often stated in RQM that "a measurement of the position of a particle, such as an electron, if carried out with greater precision than the Compton wavelength, would lead to pair production" [26]. This phenomenon is related to our reasoning behind Eq.(3) that for such an electron the uncertainty of energy is larger than the gap. It is clear, however, that the pairs created in this way can only be virtual, otherwise their recombination would lead to the production of energy out of nothing. It appears that the virtual carriers could be observed, for example, in screening effects.

In our theoretical treatment we used two simplifications. The first is the use of the three-level model of band structure. This model is accurate near the band edges of NGS and it is valid up to the inflection point on the $E(k)$ curve [27]. Secondly, we assumed that $p_{x}=p_{y}=0$ in the calculation of ZB and of the smearing. This was done for technical reasons, the difficulty being that the degeneracy of the valence $\Gamma_{8}$ bands couples light and heavy holes. However, since the conduction band is spherical [see Eq.(1)], it is clear that our results for the direction $\mathbf{z}$ are approximately valid for the other two directions as well. The isotropy of the Darwin term in Eq.(6) confirms this conclusion. Our simplifying assumption $\Delta \gg \mathcal{E}_{g}$, although not quite justified for some materials, preserves the essential spin properties of electrons in NGS. We add that another group of NGS, namely the lead salts $\mathrm{PbTe}$,
PbSe and $\mathrm{PbS}$, is characterized by the $4 \times 4$ band Hamiltonian closely resembling the Dirac Hamiltonian [2, 28]. The only complication is that the bands are ellipsoidal, which means that we deal with two maximum velocities, one for the $\mathbf{x}$ and $\mathbf{y}$ and another one for the $\mathbf{z}$ direction.

To summarize, we used the band structure of narrow gap semiconductors to show that, even in absence of external fields, semiconductor electrons experience the Zitterbewegung (trembling motion) with the characteristic frequency of $\omega_{Z}=\mathcal{E}_{g} / \hbar$ and the amplitude of $\lambda_{Z}=\hbar / m_{0}^{*} u$. If the electrons are described by twocomponent spinors for a given energy band (which is usually the case) there is no ZB but the electrons should be treated as objects of size $\lambda_{Z}$. The magnitude of $\lambda_{Z}$ in NGS can be as large as $70 \AA$. Observable consequences of these predictions are divided into those related to the final electron size and those resulting from the electron confinement to dimensions smaller than $\lambda_{Z}$. Such observations would lead to deeper understanding of electrons in solids, but they would also shed light on some still unobserved predictions of relativistic quantum mechanics and quantum field theory for electrons in vacuum.

Acknowledgments: I am pleased to thank Dr T.M. Rusin, Dr P. Janiszewski and Dr P. Pfeffer for elucidating discussions. This work was supported in part by The Polish Ministry of Sciences, Grant No PBZ-MIN008/PO3/2003.
[1] W. Zawadzki and B. Lax, Phys. Rev. Lett. 16, 1001 (1966).

[2] A.G. Aronov and G.E. Pikus, Zh. Eksp. Teor. Fiz. 51, 281 (1966); [Soviet Phys. JETP 24, 188 (1967)].

[3] W. Zawadzki, in Optical Properties of Solids, Edited by E.D. Heidemenakis (Gordon and Breach, New York, 1970), p.179.

[4] W. Zawadzki, in High Magnetic Fields in the Physics of Semiconductors II, Edited by G. Landwehr and W. Ossau (World Scientific, Singapore, 1997), p.755.

[5] W. Zawadzki, S. Klahn and U. Merkt, Phys. Rev. Lett. 55, 983 (1985).

[6] E.O. Kane, J. Phys. Chem. Solids 1, 249 (1957).

[7] S.R. Bowers and Y. Yafet, Phys. Rev. 115, 1165 (1959); W. Zawadzki, in Narrow Gap Semiconductors. Physics and Applications, Edited by W. Zawadzki (Springer, Berlin, 1980), p.85.

[8] P.A.M. Dirac, The Principles of Quantum Mechanics (Clarendon Press, Oxford, 1958).

[9] E. Schroedinger, Sitzungsber. Preuss. Acad. Wiss. Phys.Math. Kl. 24, 418 (1930). Schroedinger's calculation of ZB is reproduced in Ref. 10.

[10] A.O. Barut and A.J. Bracken, Phys. Rev. D23, 2454 (1981).

[11] W. Greiner, Relativistic Quantum Mechanics (Springer, Berlin, 1994).

[12] A. Akhiezer and V.B. Berestetskii, Quantum Electrodynamics (Nauka, Moscow, 1969). In Russian.

[13] K.T. Hecht, Quantum Mechanics (Springer, New York,
2000).

[14] R. Winkler, Physica E, 22, 450 (2004), published recently a similar expansion in a different context.

[15] W. Zawadzki, arXiv: quant-ph/0408065 v1 (2004).

[16] L.L. Foldy and S.A. Wouthuysen, Phys. Rev. 78, 29 (1950). The RHS of Eq.(21) in this paper should be completed by a factor of $1 / 2$, see Ref. 17 and our Eq.(9).

[17] M.E. Rose, Relativistic Electron Theory (Wiley, New York, 1961).

[18] J.M. Luttinger and W. Kohn, Phys. Rev. 97, 869 (1955).

[19] J. Schwinger, Phys. Rev. 82, 664 (1951).

[20] W. Greiner and J. Reinhardt, Quantum Electrodynamics, Second Edition (Springer, Berlin, 1992).

[21] E.O. Kane and E.I. Blount, in Tunneling Phenomena in Solids, Edited by E. Burstein and S. Lundqvist (Plenum Press, New York, 1969), p.79.

[22] G.H. Parker and C.A. Mead, Phys. Rev. Lett. 21, 605 (1968).

[23] F.A. Padovani and R.S. Stratton, Phys. Rev. Lett. 16, 1202 (1966).

[24] J.W. Conley and G.D. Mahan, Phys. Rev. 161, 681 (1967).

[25] A. Messiah, Mécanique quantique (Dunod, Paris, 1995).

[26] T.D. Newton and P. Wigner, Rev. Mod. Phys. 21, 400 (1949).

[27] W. Zawadzki, Adv. Phys. 23, 435 (1974).

[28] D.L. Mitchell and R.F. Wallis, Phys. Rev. 151, 581 (1965). 